\title{
Orthopäde
}

\section{Die degenerative HWS}

D ie $\mathrm{H}$ alswirbelsäule wird neben der L endenwirbelsäule häufig von degenerativen Veränderungen betroffen. Die Überalterung unserer Bevölkerung bringt es mit sich, daß diese Probleme in der Praxis fast täglich anzutreffen sind. $D$ as therapeutische $C$ redo hat sich vom weitgehenden Nihilismus vor einigen Jahren bis zum teilweise aktuell vorhandenen „overtreatment“ ausgedehnt. D ie sorgfältig ausgesuchten A rtikel der vorliegenden N ummer zeigen, daß wir jedoch weit entfernt sind von einer einheitlichen Auffassung der therapeutischen M odalitäten und daß, wie häufig in der Wirbelsäulenchirurgie, mehrere Wege zum Ziele führen können. Voraussetzung für ein effizientes therapeutisches Vorgehen bildet jedoch, hier wohl mehr als anderswo, die profunde K enntnis der G rundlagen. Von T. O hwada und M itarbeitern werden diese zu B eginn der A rtikelreihe ausführlich dargelegt. Der Ü bergang zum klinischen Teil gleichsam als A nwendung des Wissens im täglichen Leben wird von J. D vorak mit der klinischen U ntersuchung und von J. Hodler mit der B esprechung der Bildgebung erfüllt. $D$ aß auch die konservative Therapie durchaus den berechtigten Platz im $\mathrm{H}$ auptthema behauptet, legen die A rtikel von W.Schneider und J.G rifka dar. D er U mfang der restlichen B eiträge demonstriert aber deutlich die Dominanz der operativen Möglichkeiten, die heute im Bereiche der Behandlung der degenerativen Veränderungen der $\mathrm{H}$ alswirbelsäule gegeben sind. D ie M annigfaltigkeit der grundsätzlichen A uffassungen und der dargestellten Techniken illustrieren lebhaft die nach wie vor im $\mathrm{G}$ ang befindlichen Kontroversen über die „I dealtherapie“. Im W issen, daß die vorliegende $A$ usgabe des $O$ rthopäden kein abschließendes $B$ ild über die verschiedenen anstehenden Probleme zu geben vermag, war es die $A$ bsicht, mit A uswahl von kompetenten A utoren, einen D iskussionsbeitrag zu einem komplexen Thema zu leisten.

D. Grob

J.D vorak

N.G schwend

$D$ ie H erausgeber 